\title{
Uso das tecnologias da informação em grupos de pesquisa brasileiros: revisão de teses e dissertações
}

Use of information technologies in Brazilian research groups: review of theses and dissertations

\author{
Uso de tecnologías de la información en grupos de \\ investigación brasileños: revisión de tesis y disertaciones
}

\author{
Elaine Pasqualini' \\ Sandra Regina \\ Gimeniz-Paschoal ${ }^{2}$ \\ Rosemeiry Castro Prado ${ }^{3}$
}

\begin{abstract}
${ }^{1}$ Pós-doutora em Educação pela Universidade Estadual Paulista. Professora dos cursos de Análise e Desenvolvimento de Sistemas e Segurança da Informação da Faculdade de Tecnologia de Ourinhos. Ourinhos, São Paulo, Brasil. Orcid: https://orcid.org/0000-0002-7758-1419. E-mail: elaine.pasqualini@fatec.sp.gov.br

${ }^{2}$ Doutora em Psicologia pela Universidade de São Paulo e livre-docente em Psicologia do Desenvolvimento. Professora do Programa de PósGraduação em Educação da Unesp. Marília, São Paulo, Brasil. Orcid: https://orcid.org/0000-0003-0330-132X. E-mail: srg.paschoal@unesp.br

${ }^{3}$ Doutora em Educação Matemática pela Universidade Estadual Paulista. Coordenadora do curso de Ciência de Dados da Faculdade de Tecnologia de Ourinhos. Ourinhos, São Paulo, Brasil. Orcid: https://orcid.org/00000002-6541-1607. E-mail: rose.prado@fatecourinhos.edu.br
\end{abstract}

Recebido para publicação em: 25.5.2021

Aprovado em: 26.7.2021

\section{Resumo}

O objetivo deste trabalho foi investigar teses e dissertações sobre o uso das tecnologias da informação em grupos de pesquisa. Foi realizado um levantamento na Biblioteca Digital de Teses e Dissertações, sendo encontradas 12 (70,59\%) dissertações e cinco $(29,41 \%)$ teses. Os resultados mostraram que os pesquisadores vêm sendo influenciados pelas tecnologias, criando modelos e usando ferramentas tecnológicas para organizar suas atividades. Concluiu-se que houve escassez de estudos, mas aqueles encontrados trouxeram informações que podem ser usadas para gerenciar as atividades em grupos de pesquisa.

Palavras-chave: grupos de pesquisa; tecnologia da informação; revisão.

\section{Abstract}

The aim of this work was to investigate theses and dissertations on the use of information technologies in research groups. A survey was carried out in the Digital Library of Theses and Dissertations, having found 12 (70.59\%) dissertations and five $(29.41 \%)$. The results showed that researchers have been influenced by technologies, creating models, and using technological tools to organize their 
activities. In conclusion, there was a shortage of studies, but those found brought information that can be used to manage the activities in research groups.

Keywords: research groups; information technology; review.

\section{Resumen}

El objetivo de este trabajo fue la investigación de tesis y disertaciones sobre el uso de las tecnologías de la información en grupos de investigación. Se realizó una busca en la Biblioteca Digital de Tesis y Disertaciones, encontrándose 12 $(70,59 \%)$ disertaciones y cinco $(29,41 \%)$ tesis. Los resultados mostraron que los investigadores han sido influenciados por las tecnologías, creando modelos y utilizando herramientas tecnológicas para organizar sus actividades. Se concluyó que hubo escasez de estudios, pero los encontrados aportaron información que se puede utilizar para gestionar las actividades en los grupos de investigación.

Palabras clave: grupos de investigación; tecnología de la información; revisión.

\section{Introdução}

O objetivo principal deste trabalho foi investigar teses e dissertações sobre o uso das tecnologias da informação em grupos de pesquisa em instituições de ensino superior do Brasil. Este trabalho faz parte de um estudo para a criação e avaliação de um sistema (software) para gerenciar atividades ligadas a grupos de pesquisas. Dessa forma, antes de sua construção, fez-se necessária essa investigação.

Assim, é importante elaborar uma breve explanação e um rápido histórico sobre pós-graduação e grupos de pesquisa. No Brasil, a partir da década de 1960, ocorreu a determinação da diferenciação de cursos de pós-graduação em stricto sensu e lato sensu (PILATTI, 2006). O surgimento da primeira pós-graduação stricto sensu no Brasil se deu em 1965, na Pontifícia Universidade Católica do Rio de Janeiro, em nível de mestrado, na área da educação, e exigia atividades de pesquisa e produção de conhecimento (MOTTA, 2014).

Com o crescimento do número de cursos de pós-graduação stricto sensu, os grupos de pesquisa começaram a se intensificar, constituindo-se por pessoas com diferentes níveis de formação, centrados em uma liderança com experiência na produção de conhecimentos científicos e sendo operacionalizados por projetos de pesquisa de acordo com as linhas de estudo de cada grupo (ERDMANN; MELLO; ANDRADE; KLOCK, 2010).

Segundo Figueira Netto (1994), os primeiros trabalhos sobre o tema surgiram na década de 1990, na maioria como produto de dissertações e teses. Em sua pesquisa a respeito do uso da internet por comunidades acadêmico-científicas no Brasil, o autor confirmou que um dos benefícios das redes eletrônicas era a comunicação entre os pesquisadores. Ferreira (1995) evidenciou as necessidades de informação 
de uma comunidade universitária e concluiu que as redes possibilitaram uma nova forma de comunicação entre pesquisadores, docentes e alunos.

As TICs

representam

um papel

cada vez mais

importante

no ambiente

de pesquisa

A partir da década de 1990, os estudos brasileiros sobre a internet e as tecnologias se tornaram mais presentes na literatura, apontando o correio eletrônico como o recurso mais utilizado pelos pesquisadores, beneficiando a troca de informações (CASTELLANI; REINHARD; ZWICKER, 1998). Atualmente, tem-se outras tecnologias vinculadas, como videoconferências, fóruns, que podem ser combinadas para potencializar a comunicação e o aprendizado (COELHO NETO; BLANCO; ARAÚJO, 2019).

Todos esses recursos dizem respeito às tecnologias da informação e comunicação (TIC) que tratam de procedimentos e equipamentos usados para processar a informação e comunicá-la aos interessados (CORREIA; SANTOS, 2013). As TICs representam um papel cada vez mais importante no ambiente de pesquisa, pois podem facilitar o modo de interação entre os membros de um grupo de pesquisa ao viabilizar a exploração de recursos que permitem a comunicação em qualquer hora, independentemente do lugar.

Para Lemos, Silva, Silva e Lima (2019), a aproximação discente da teoria, da prática e da pesquisa, bem como de seus procedimentos sistemáticos de produção científica e disseminação do conhecimento, é um caminho adequado para a aprendizagem.

Nesse contexto em que a prática do compartilhamento de conhecimento é imprescindível, o presente estudo busca contribuir para suscitar reflexões sobre o uso dessas tecnologias nos grupos de pesquisa.

\section{Metodologia}

Para atingir o objetivo da pesquisa, foi realizada uma revisão sistemática para responder ao seguinte questionamento: quais tecnologias e ferramentas são usadas pelos grupos de pesquisas para organizar suas atividades e/ou que benefícios trouxeram caso tenham sido especificados nos trabalhos encontrados?

A revisão sistemática é considerada um passo inicial para qualquer pesquisa, a fim de se descobrir o que existe na literatura sobre o assunto a ser investigado (CONFORTO; AMARAL; SILVA, 2011). As fontes primárias podem ser de bases de dados, sendo definidos os descritores, compostos por palavras-chave referentes à pesquisa, e critérios de inclusão/exclusão (FERENHOF; FERNANDES, 2016).

Foi utilizada a base da Biblioteca Digital de Teses e Dissertações (BDTD), pelo fato de os trabalhos de doutorado e mestrado estarem vinculados às instituições de ensino superior e, desse modo, terem grupos de pesquisa envolvidos.

Foi usado o descritor "grupos de pesquisa", encontrando-se mais de 8 mil trabalhos. Refinou-se a busca combinando-se as palavras "sistema", "software" e "tecnologias da informação", sem outros filtros, obtendo-se redução de mais da metade dos trabalhos encontrados. 
Foram então lidos os títulos e os resumos. Se o trabalho não fazia parte do escopo desta pesquisa, por não ser ligado a instituições de ensino superior do Brasil ou apenas mencionar grupos de pesquisa sem a descrição do uso de tecnologias da informação como meio de comunicação no grupo ou entre grupos de pesquisa, era excluído. Se o trabalho se enquadrava no que buscávamos, era armazenado no computador para ser analisado depois do processo de busca ser concluído. Todo o levantamento foi realizado por dois dos autores e houve concordância em relação aos trabalhos levantados, que então foram lidos na íntegra.

\section{Resultados e discussão}

Foram identificados 17 trabalhos. Considerando que o número de grupos de pesquisa cadastrados no Censo de 2016 pelo Conselho Nacional de Pesquisa (CNPq) é de 37.640 (CONSELHO NACIONAL DE DESENVOLVIMENTO CIENTÍFICO E TECNOLÓGICO, 2020), verifica-se que é pequena quantidade de estudos encontrados. Porém, deve-se ressaltar que os grupos estão voltados para suas linhas de pesquisa, com suas produções a elas vinculadas, ou seja, podem usar as tecnologias, mas não torná-las objeto de investigação.

No Quadro 1, constam os 17 trabalhos, de acordo com o tipo: dissertação (D)/tese (T), o autor e o ano, o nome da instituição e a área de concentração.

\section{Quadro 1 - Trabalhos encontrados, de acordo com o tipo, autor e ano, nome da instituição e área de concentração $(\mathrm{N}=17)$.}

\begin{tabular}{|l|l|l|l|}
\hline Tipo & Autor, ano & Instituição & Área \\
\hline T & Souza, 1999 & Universidade de Brasília (UnB) & Ciência da informação \\
\hline D & $\begin{array}{l}\text { Santos Junior, } \\
2000\end{array}$ & $\begin{array}{l}\text { Universidade Federal do Rio Grande do Sul } \\
\text { (UFRGS) }\end{array}$ & Sociologia \\
\hline D & Lichtnow, 2001 & $\begin{array}{l}\text { Universidade Federal de Santa Catarina } \\
\text { (UFSC) }\end{array}$ & Ciência da computação \\
\hline T & $\begin{array}{l}\text { Freitas Junior, } \\
\text { 2003 }\end{array}$ & $\begin{array}{l}\text { Universidade Federal de Santa Catarina } \\
\text { (UFSC) }\end{array}$ & Engenharia \\
\hline D & Alves, 2004 & $\begin{array}{l}\text { Universidade Federal do Rio Grande do Sul } \\
\text { (UFRGS) }\end{array}$ & Ciência da informação \\
\hline D & Lima, 2005 & Universidade Federal de São Carlos (UFSCar) & Engenharia \\
\hline D & Ramos, 2006 & $\begin{array}{l}\text { Pontifícia Universidade Católica de Campinas } \\
\text { (PUC-Campinas) }\end{array}$ & Ciência da informação \\
\hline D & Santos, 2007 & Universidade Presbiteriana Mackenzie (UPM) & Engenharia \\
\hline T & Miranda, 2008 & $\begin{array}{l}\text { Pontifícia Universidade Católica de São Paulo } \\
\text { (PUC-SP) }\end{array}$ & Educação \\
\hline D & Santos, 2010 & Universidade de São Paulo (USP) & Engenharia \\
\hline D & Felix, 2011 & Universidade de São Paulo (USP) & Comunicações e artes \\
\hline
\end{tabular}


(continuação)

\begin{tabular}{|l|l|l|l|}
\hline D & Ferraz, 2011 & Universidade Federal de Pernambuco (UFPE) & Administração \\
\hline D & Ferrasi, 2013 & Universidade Estadual Paulista (UNESP) & Biotecnologia médica \\
\hline D & Tisott, 2015 & Universidade de Caxias do Sul (UCS) & Administração \\
\hline T & Santos, 2016 & Universidade de São Paulo (USP) & Engenharia \\
\hline D & Dutra, 2017 & Universidade Federal de Santa Maria (UFSM) & Engenharia \\
\hline T & Sampaio, 2017 & Universidade Federal da Bahia (UFBA) & Geologia \\
\hline
\end{tabular}

Nota: D - dissertação; T - tese.

Fonte: Elaborado pelos autores.

Dos trabalhos considerados, observa-se que $12(70,59 \%)$ eram de mestrado e cinco $(29,41 \%)$ de doutorado. De 1999 a 2004 foram encontrados cinco trabalhos $(29,41 \%)$, de 2005 a 2010 outros cinco $(29,41 \%)$ e de 2011 a 2017 foram sete $(41,18 \%)$.

Com relação às instituições de ensino superior, a PUC teve dois $(11,76 \%)$ trabalhos identificados, a USP três (17,65\%), a UNESP um (5,88\%), a UFRGS dois (11,76\%), a UFPE um (5,88\%), a UPM um (5,88\%), a UFBA um (5,88\%), a UCS um $(5,88 \%)$, a UFSM um (5,88\%), a UFSC dois (11,76\%), a UFSCar um (5,88\%) e a UnB um (5,88\%). Dessa forma, quatro $(23,53 \%)$ instituições privadas e 13 (76,47\%) públicas.

Quanto às áreas, a da ciência da informação foi representada por três trabalhos $(17,65 \%)$, a engenharia por seis $(35,2 \%)$, a administração teve dois $(11,77 \%)$, as áreas de comunicação e artes também tiveram dois trabalhos (5,88\%), e os campos da geologia, educação, ciência da computação, sociologia e biotecnologia médica tiveram um cada $(5,88 \% \times 5)$. Os cursos de geologia e ciências da computação $(11,76 \%)$ estão relacionados à área das ciências exatas e da Terra; o de engenharia pertence ao campo das engenharias (35,29\%); os de administração e de ciência da informação $(29,43 \%)$ integram as ciências sociais aplicadas; os de sociologia e de educação $(11,76 \%)$ pertencem às ciências humanas; o curso de comunicação e artes (5,88\%) integra o campo da linguística, das letras e da artes; e o de biotecnologia médica $(5,88 \%)$ se relaciona à área de conhecimento multidisciplinar. A predominância da área das engenharias pode dizer respeito ao fato de que o ensino do campo tem passado por discussões e reformulações decorrentes do impacto do conhecimento tecnológico, resultando em propostas para novas pedagogia e metodologia (AZAMBUJA, 2013).

Com relação aos objetivos, participantes, instrumentos e resultados das teses e dissertações investigados neste trabalho, a seguir são apresentadas nossas considerações.

A tese de Souza (1999) teve como objetivo verificar se o uso das tecnologias no processo de comunicação dos pesquisadores da Empresa Brasileira de Pesquisa Agropecuária (Embrapa) contribuiu para modificar as estruturas e estratégias do modelo de produção, legitimação e difusão do conhecimento. Foi utilizado um questionário enviado por e-mail com questões abertas e fechadas para os pesquisadores da instituição em diferentes unidades, selecionados de maneira aleatória. O uso mensal 
das tecnologias de informação apresentou os seguintes percentuais de utilização entre os participantes: reuniões em meio eletrônico, 25,3\%; troca de ideias entre pesquisadores, 26,7\%; busca na literatura, 32\%; intercâmbio de documentos, 29,3\%; conferência eletrônica, 17,3\%; avaliação de trabalhos científicos on-line, 40\%, entre outros com menor relevância quantitativa. Dessa forma, as tecnologias usadas contribuíram para a produção e divulgação do conhecimento, segundo o autor.

0 trabalho de Lichtnow teve

o propósito de facilitar 0 compartilhamento de conhecimentos e informações dentro de um grupo de pesquisa, por meio de um protótipo
Santos Júnior (2000) analisou a organização e funcionamento de grupos de pesquisa, incluindo as práticas interativas. Um questionário com questões fechadas foi enviado por e-mail e entrevistas semiestruturadas foram feitas com os líderes de grupos da UFRGS. As práticas internas e externas (a relação de um grupo com outros grupos de outras instituições) dos grupos utilizavam como meio de comunicação os e-mails, 39\% para as internas e $58 \%$ para as externas. Nesse trabalho foi citada a falta de um sistema e de outras ferramentas para integrar as informações e gerenciar a comunicação e a interação de grupos de pesquisa, que são compostos por membros permanentes e temporários.

O trabalho de Lichtnow (2001) teve o propósito de facilitar o compartilhamento de conhecimentos e informações dentro de um grupo de pesquisa, por meio de um protótipo desenvolvido para um projeto que envolve a Universidade Federal de Santa Catarina, a Universidade do Alto Vale de Itajaí, a Universidade do Extremo Sul de Santa Catarina e a Universidade de Kaiserlautern, na Alemanha. Realizou-se uma revisão bibliográfica e entrevistas com a coordenação do projeto, a fim de identificar as necessidades e funcionalidades do protótipo. Como resultado, várias funcionalidades foram sugeridas, como cadastro de projetos, biblioteca, mapas de conhecimento ou competência, tecnologias groupware, ou seja, usadas para ajudar pessoas a trabalharem em grupo, como e-mail, groups calendars (escalonamento de tarefas entre as pessoas), collaborative writing systems (utilizados para que cada integrante possa participar da autoria de um documento e acompanhar as alterações realizadas), entre outras. As ferramentas sugeridas poderiam trazer benefícios ao grupo, permitindo o conhecimento dos projetos e de cada membro de grupo, identificando as atividades de cada um.

A tese de Freitas Júnior (2003) propôs um modelo de sistema de gestão do conhecimento para gerenciar a criação, a disseminação e o compartilhamento de conhecimentos para grupos de pesquisa. Participaram membros do grupo Stela, da Universidade Federal de Santa Catarina. Foi feita a revisão da literatura e aplicado um questionário com perguntas abertas e fechadas. Como resultado, foi proposta a criação de um portal integrado para grupos de pesquisa, contendo: ferramentas para fazer uso de biblioteca digital, e-mail, videoconferência, notificação de avisos, FAQ, armazenamento digital de projetos de pesquisa do grupo, links relevantes para o grupo de pesquisa, lições aprendidas (armazenamento de soluções sobre como realizar determinadas tarefas elaboradas pelo grupo), calendário de eventos, lista 
de discussão, mapas de competência (indicação de áreas de interesse e nível de conhecimento de cada pesquisador) etc.

O trabalho de Alves (2004) teve como objetivo analisar a conceituação de comunidades científicas, bem como suas características, reconfiguradas pela internet e pelas possibilidades de interatividade entre os pesquisadores. Os participantes eram compostos por quatro grupos de pesquisadores na área de biofarmacologia. Foram aplicados um questionário com perguntas abertas e fechadas, enviado por e-mail, e entrevistas. O trabalho não apresentou uma análise estatística dos resultados e descreveu que foram usados e-mails e chats para a comunicação dos grupos, socializando as informações e a mobilidade dos dados. Destaca-se que os participantes, já naquele ano da pesquisa, relataram que gostariam que fosse criado um ambiente virtual que integrasse várias ferramentas de comunicação e de trabalho colaborativo. Lima (2005) descreveu a experiência de implantação de um software de apoio à gestão do conhecimento em uma rede de pesquisa. Os participantes pertenciam a um grupo de pesquisa e foram definidos por meio de uma amostra estratificada proporcional. Como instrumentos, foram utilizadas a pesquisa bibliográfica e a combinação de pesquisa de campo, na descrição da implantação, e entrevista, na identificação das práticas e necessidades do grupo. Como resultado, os seguintes requisitos do sistema foram apontados: cadastro e acesso de membros, lista de conhecimentos dos usuários, cadastro/consulta de documentos do grupo, mecanismos de busca e gerenciamento de conteúdo, e-mails, customização de cores e fontes, fórum, chat, agenda, calendário, estatísticas sobre o site (por usuário, assunto, contribuição), entre outros.

A dissertação de Ramos (2006) analisou qual era a efetiva utilização de sistemas de informação para o apoio ao trabalho colaborativo em grupos de pesquisa. Participaram líderes de grupo cadastrados no CNPq que foram atualizados nos últimos 12 meses, usando amostragem probabilística aleatória de cada grande área do conhecimento. Foi utilizado um questionário com perguntas abertas e fechadas,

Os resultados

mostraram que $68 \%$ utilizavam

o computador

para se comunicar

com os demais

pesquisadores do

grupo, sendo que

$32 \%$ usavam o

ambiente virtual

colaborativo enviado por e-mail. Os resultados mostraram que $68 \%$ dos participantes utilizavam o computador para se comunicar com os demais pesquisadores/membros do grupo, sendo que 32\% usavam o ambiente virtual colaborativo (AVC). Desse montante, apenas 16\% especificaram corretamente o termo AVC, enquanto 59\% descreveram ser e-mail, 13\% citaram AVC como AVA (Ambiente Virtual de Aprendizagem) e 13\% mencionaram outras ferramentas.

O objetivo da dissertação de Santos (2007) foi adaptar um sistema já existente para atender às necessidades de recuperação e gestão de documentos de grupos de pesquisa, especificamente o CRAAM (Centro de Radioastronomia e Astrofísica Mackenzie). Para isso foram usadas as ferramentas de linguagem Java MySQL, Servlets e JavaServer Pages. Como resultado, elaborou-se um sistema para ser usado em ambientes de gerenciamento de documentos para grupos de pesquisa. 
A tese de Miranda (2008) analisou o processo de inserção do uso de ambientes virtuais do Grupo de Estudos e Pesquisa em Interdisciplinaridade (GEPI). Usou-se o AVA Teleduc, que foi configurado para os integrantes. Dessa forma, pôde-se registrar o histórico e ações do grupo, promover o acesso dos integrantes, enviar e-mails, disponibilizar materiais de estudo, fazer enquetes, alterar perfil, consultar agenda, fórum etc. Um dos resultados encontrados foi a dificuldade de diálogo entre os integrantes usando o ambiente virtual.

A dissertação de Santos (2010) propôs desenvolver programas de computador que atuassem como ferramentas para auxiliar pesquisadores durante os processos de pesquisa e criação de conhecimento. Foram usadas as ferramentas Apache Jackrabbit, JavaServer Faces e Java Content Repository. Dessa forma, foi criado um repositório para armazenar conteúdos coletados por pesquisadores durante o processo de pesquisa e uma ferramenta para acessar e manipular o repositório, de modo que todo conteúdo científico pudesse ser acessado e compartilhado pelos pesquisadores. Observou-se que apenas a introdução de tecnologias da informação não resolve todos os problemas de comunicação. Então sugeriu-se a implantação de técnicas de gestão do conhecimento.

Observou-se

que apenas a introdução de tecnologias da informação não resolve todos os problemas de comunicação
Em sua dissertação, Felix (2011) se propôs a criar um portal para armazenar o histórico do Grupo Cibernética Pedagógica da USP. Foram realizadas uma revisão da literatura e entrevistas com os envolvidos, a fim de identificar as necessidades do portal. Para a implementação, empregou-se as linguagens PHP e HTML. Foram criadas funcionalidades como recursos de busca e pesquisa, links para outros grupos com a mesma temática, contatos, história do grupo, projetos em andamento e finalizados, textos relacionados aos assuntos de interesse do grupo e "fale conosco". O trabalho apresentou recursos importantes para os integrantes, mas alguns não foram desenvolvidos, como o armazenamento da produção do grupo, ferramenta fundamental por representar a essência de trabalhos e conhecimentos (ERDMANN; MELLO; ANDRADE; KLOCK, 2010).

Ferraz (2011), em sua dissertação, teve como objetivo inventariar e enquadrar os grupos de pesquisa na condição de comunidades virtuais de prática e evidenciar os mecanismos e os recursos compartilhados. Foi elaborado um questionário com questões objetivas, enviado por e-mail, e entrevistas semiestruturadas feitas com os líderes de grupos de pesquisa da UFPE. Quase todos os grupos (97,5\%) faziam uso de e-mail, mais da metade $(61,7 \%)$ utilizava softwares de auxílio à pesquisa (não foram descritos quais), home page do grupo (37\%), chat (30,9\%), fórum de discussão (19,8\%), blogs (12,3\%), redes sociais, como Facebook e Twitter (9,9\%), wikis $(6,2 \%)$ e ambiente virtual (2,5\%). Nesse trabalho, várias tecnologias foram usadas, porém sem integração de dados, o que pode gerar problemas como a perda e a desconexão das informações (JANNUZZI; FALSARELLA; SUGAHARA, 2014).

Na pesquisa de Ferrasi (2013), o propósito foi desenvolver um software para o gerenciamento de dados gerados a partir de projetos na área de ciências da saúde. Para a 
criação, foi realizada uma análise de requisitos, usada a linguagem de programação Java, banco de dados Firebird, Apache Tomcat, JasperRepots, Hibernate e PrimeFaces. O sistema possibilitou o acesso aos participantes por meio de login e senha, permitiu o cadastro de projetos de acordo com a área envolvida e acesso aos dados de pesquisa, proporcionando interação entre diferentes grupos.

O trabalho de Tisott (2015) analisou a dinâmica dos grupos de pesquisa em nanotecnologia do CNPq. Os líderes e integrantes de grupos da área responderam a questionários com perguntas abertas e fechadas. Como resultado, percebeu-se que os participantes faziam uso das tecnologias da informação, como e-mail, fórum etc., ou seja, os grupos possuíam uma configuração voltada ao compartilhamento do conhecimento, sendo necessária a implantação da gestão de conhecimento.

Santos (2016), em seu trabalho de doutorado, investigou em que medida as práticas de gestão do conhecimento associadas às tecno-

Os grupos possuíam uma configuração voltada ao compartillhamento do conhecimento, sendo necessária a implantação da gestão de conhecimento logias de computação em nuvem poderiam contribuir para a produção em ambientes de pesquisa. Os participantes foram estudantes de pós-graduação, pesquisadores, mestres, doutores e professores das áreas de computação e engenharia de universidades públicas no Brasil. Usou-se amostragem não probabilística e um questionário eletrônico com questões objetivas. Como resultado, $24,32 \%$ dos respondentes disseram que, em suas pesquisas, acessavam regularmente uma VPN (virtual private network, rede de comunicação privada, em português) e 27,41\% usavam algum tipo de computação em nuvem. 0 autor destaca ser necessário que os membros dos grupos de pesquisa compreendam a importância de gerenciar o conhecimento científico, de modo que possam acessar, adaptar, criar, compartilhar e usar as informações disponíveis nas nuvens, produzidas pelas tecnologias.

No trabalho de Dutra (2017), o objetivo foi elaborar um modelo para o desenvolvimento de projetos de pesquisa do Laboratório de Projeto de Sistemas Técnicos (LPST), da Universidade Federal de Santa Maria. O grupo respondeu a um questionário pela internet e foram usadas, como instrumentos, observações, entrevistas informais e pesquisas documentais. Os resultados apontaram o uso de algumas tecnologias, como e-mails, redes sociais e diversos aplicativos para comunicação, ou seja, ferramentas contemporâneas. Porém, para melhorar o desenvolvimento dos projetos, com base nos problemas investigados foi criado um modelo de gerenciamento.

Sampaio (2017) buscou identificar ferramentas computacionais para auxiliar na colaboração e nas pesquisas em grupos de estudos geocientíficos. Participaram os coordenadores do Instituto Nacional de Ciências e Tecnologia e do Laboratório de Estudos Costeiros. Foram realizadas a revisão da literatura e reuniões. Os participantes destacaram os canais de entrega de conteúdo digital (e-mail, WebSyndication), ferramentas de computação em nuvem (DropBox, Google Drive, OwnCloud), sistemas gerenciadores de conteúdo (WordPress), GIS (sistemas de informação 
geográfica), globos virtuais (Microsoft Virtual Earth, Google Maps, entre outros mais especializados), sistemas que permitem agendamentos de encontros e eventos (Doodle), softwares que possibilitam comunicação pela internet por meio de conexões de voz, vídeo e texto (Skype, WhatsApp, Telegram) e sistemas que proporcionam o gerenciamento de projetos (Microsoft Project, Redmine) etc. Nesse trabalho foram, novamente, identificadas a utilização de várias tecnologias, mas sem a integração entre elas, o que pode ocasionar perdas de dados, contrapondo-se à possibilidade de proporcionar informações combinadas e estruturadas.

A Tabela 1 apresenta as tecnologias e ferramentas usadas em grupos de pesquisa.

Tabela 1 - Tecnologias e ferramentas utilizadas em grupos de pesquisa, de acordo com os autores, ano, frequência (f) e porcentagem (\%) $(\mathrm{N}=17)$.

\begin{tabular}{l|l|l|l} 
Tecnologias e ferramentas & Autor, ano & $\mathbf{f}$ & $\%$ \\
\hline E-mails & $\begin{array}{l}\text { Alves, 2004; Dutra, 2017; Ferraz, 2011; } \\
\text { Ramos, 2006; Sampaio, 2017; Santos } \\
\text { Junior, 2000; Tisot, 2015 }\end{array}$ & 7 & 41,18 \\
\hline $\begin{array}{l}\text { Criação de portais, home pages e } \\
\text { sistemas em geral }\end{array}$ & $\begin{array}{l}\text { Felix, 2011; Freitas Junior, 2003; Ferraz, } \\
\text { 2011; Ferrasi, 2013; Lima, 2005 }\end{array}$ & 5 & 29,41 \\
\hline Ambientes virtuais de aprendizagem & Ferraz, 2011; Miranda, 2008; Ramos, 2006 & 3 & 17,65 \\
\hline Ferramentas para gerenciamento de \\
projetos e de documentos & Sampaio, 2017; Santos, 2007 & 2 & 11,76 \\
\hline Tecnologias groupware & & 2 & \\
\hline Redes sociais & Lichtnow, 2001; Sampaio, 2017 & 2 & 11,76 \\
\hline Aplicativos em geral & Dutra, 2017; Ferraz, 2011 & 2 & 11,76 \\
\hline Listas de discussão e fórum & Dutra, 2017; Sampaio, 2017 & 2 & 11,76 \\
\hline Blogs & Ferraz, 2011; Tisott, 2015 & 2 & 11,76 \\
\hline Chats & Ferraz, 2011 & 1 & 5,88 \\
\hline Wikis & Ferraz, 2011 & 1 & 5,88 \\
\hline Ferramentas de computação em nuvem & Sampaio, 2017 & 1 & 5,88 \\
\hline
\end{tabular}

Fonte: Elaborado pelos autores.

Destacou-se o uso do e-mail como ferramenta tecnológica presente nos grupos de pesquisa, o que pode estar relacionado ao fato de se tratar de um dos serviços mais antigos e utilizados na internet. O endereço eletrônico foi o recurso mais importante para o desenvolvimento da rede mundial de computadores, tornando-se um centro integrado de informações que oferece oportunidades para a realização de pesquisas, pois permite a comunicação por mensagens e o envio de documentos com rapidez, possibilitando a comunicação com indivíduos ou grupos distantes uns dos outros (REEDY; SCHULLO; ZIMMERMAN, 2006).

Também se mostrou relevante o uso de sistemas em geral como ferramentas de apoio aos pesquisadores. Isso pode ocorrer pelo fato de os sistemas permitirem 
coletar, armazenar, recuperar e disseminar informações para fins específicos (JANNUZZI; FALSARELLA; SUGAHARA, 2014).

$\mathrm{O}$ uso de ambientes virtuais de aprendizagem também foi verificado. Prado e colaboradores (2012) descreveram a criação de um ambiente virtual de aprendizagem em um grupo de pesquisa do curso de enfermagem da USP para o compartilhamento de informações. Foram utilizadas ferramentas como fórum, chat, lista de contato e perfil dos integrantes, atas de reuniões, biblioteca virtual e armazenamento de trabalhos aceitos em eventos e periódicos. Foram criados, ainda, espaços para as informações sobre os objetivos, as metas e as normas de participação no grupo, incluindo o registro da história do mesmo e outras ferramentas. Dessa forma, os resultados da construção de um ambiente virtual para um grupo de pesquisa vão ao encontro da literatura, que descreve a importância de um espaço para estimular as produções científıcas.

Assim, destaca-se a importância de grupos de pesquisa no aprofundamento da busca de conhecimentos, que pode se beneficiar da utilização dos meios eletrônicos e das demais alternativas disponíveis. A participação de acadêmicos nos grupos favorece uma visão ampliada do processo de pesquisa, uma vez que produz vínculo com o tema abordado e os pesquisadores que integram o grupo. Os recursos tecnológicos estão inseridos em todas as fases da pesquisa. Desde a revisão da literatura, com as buscas em bibliotecas virtuais, periódicos eletrônicos e no acervo bibliográfico do próprio grupo, até a elaboração de bancos de dados, entre outras possibilidades (KRAHL et al., 2008).

O conhecimento gerado necessita ser socializado e compartilhado por meio de interações entre grupos e também pelas tecnologias da informação. Dessa forma, o conhecimento precisa de gestão, que envolve a criação de meios para captação, manutenção, armazenagem, divulgação e aplicação do conhecimento, que pode ser obtido por ferramentas e recursos computacionais, para disponibilizar repositórios de conhecimentos (ODELIUS et al., 2011).

Portanto, a gestão do conhecimento vai além da tecnologia empregada, depende também do papel do líder do grupo de pesquisa e da mensuração de resultados, entre outras ações, para que haja interação no grupo e com colegas de outros grupos.

\section{Conclusão}

Concluiu-se que há uma escassez de estudos sobre a temática, mas os trabalhos encontrados trouxeram informações que podem suscitar novas pesquisas e reflexões, de maneira a serem usados para gerenciar e organizar as atividades em grupos de pesquisa.

Este trabalho identificou como principal necessidade o uso de ferramentas e softwares por grupos de pesquisa em instituições de ensino superior no Brasil para o compartilhamento de informações e o gerenciamento de atividades. 
Os pesquisadores elaboraram modelos e usaram ferramentas - tais como e-mails, home pages e ambientes virtuais de aprendizagem, entre outras - para coordenar e sistematizar suas atividades de estudos com o uso das tecnologias.

Percebeu-se que, ao longo dos anos, os pesquisadores vêm sendo bastante influenciados pelas tecnologias da informação e comunicação, a ponto de elaborarem programas e usarem instrumentos que caminhem ao encontro de seus propósitos e dos problemas investigados.

Vale ressaltar que nos trabalhos pesquisados não foi identificado um sistema que integrasse as diversas funções aqui apontadas, com acesso pelo administrador/ líder do grupo e demais membros, como a inserção de metas e objetivos, quadro de avisos, cadastro de disponibilidade dos usuários, inserção de biblioteca de interesse, produção do grupo, registro de eventos, cadastro de atas, aplicação de trabalho colaborativo em que cada integrante pode participar da autoria de um documento e acompanhar as alterações realizadas, entre outras.

Dessa forma, são necessárias novas pesquisas voltadas para a criação de tais sistemas, bem como a contínua busca de informações dessa natureza em diferentes bases de dados e também de maneira direta com os grupos de pesquisa.

\section{Referências}

ALVES, Sabrina Augusta de Oliveira. Comunidade científica via internet (CCI): uma análise sobre o uso de novas tecnologias digitais na pesquisa científica do Brasil (um estudo de caso com o grupo PROCAD). 2004. Dissertação (Mestrado) Faculdade de Biblioteconomia e Educação, Universidade Federal do Rio Grande do Sul, Porto Alegre, 2004.

AZAMBUJA, Marcos Jolbert Cáceres. A IPTV como modalidade de educação: um estudo de caso no ensino de Engenharia. 2013. Dissertação (Mestrado) - Escola Politécnica, Universidade de São Paulo, São Paulo, 2013.

CASTELLANI, Márcia Reiff; REINHARD, Nicolau; ZWICKER, Ronaldo. Cultura organizacional e tecnologia da informação: um estudo do uso da internet na atividade acadêmica de pesquisa. In: ENCONTRO DA ASSOCIAÇÃO NACIONAL DE PÓS-GRADUAÇÃO EM ADMINISTRAÇÃO, 23., 1998, Foz do Iguaçu. Anais [...] Rio de Janeiro: ANPAD, 1998, p. 1-15.

COELHO NETO, João; BLANCO, Marilia Bazan; ARAÚJO, Roberta Negrão de. As tecnologias da informação e comunicação para o ensino de ciências: percepções, desafios e possibilidades para o contexto educacional. Ensino de Ciências

e Tecnologia em Revista, Santo Ângelo, v. 9, n. 2. p. 3-16, maio/ago. 2019. Disponível em: http://srvapp2s.urisan.tche.br/seer/index.php/encitec/article/ view/2218/pdf-2218. Acesso em: 08 fev. 2020. 
CONFORTO, Edivandro Carlos; AMARAL, Daniel Capaldo; SILVA, Sergio Luis da. Roteiro para revisão bibliográfica sistemática: aplicação no desenvolvimento de produtos e gerenciamento de projetos. In: CONGRESSO BRASILEIRO DE GESTÃO DE DESENVOLVIMENTO DE PRODUTO, 8, 2011, Porto Alegre. Anais [...]. [S. I.: s. n.], 2011. p. 1-12.

CONSELHO NACIONAL DE DESENVOLVIMENTO CIENTÍFICO E TECNOLÓGICO. Censo atual. Brasília, DF: CNPq, [202-]. Disponível em: http://lattes.cnpq.br/web/ dgp/censo-atual/. Acesso em: 16 nov. 2020.

CORREIA, Rosangela Linhares.; SANTOS, José Gonçalo dos. A importância da tecnologia da informação e comunicação (TIC) na educação a distância (EAD) do ensino superior (IES). Revista Aprendizagem em EAD, [Brasília, DF], v. 2, p. 1-16, nov. 2013.

DUTRA, Marianna Caroline Zanini. Modelo de gestão integrada para o desenvolvimento de projetos em um grupo de pesquisa. 2017. Dissertação (Mestrado) - Departamento de Engenharia de Produção, Universidade Federal de Santa Maria, Santa Maria, 2017.

ERDMANN, Alacoque Lorenzini; MELLO, Ana Lúcia Ferreira de; ANDRADE, Selma Regina de; KLOCK, Patrícia. Funcionalidade dos grupos de pesquisa de administração/gestão/ gerência de enfermagem. Rev Rene, Fortaleza, v. 11, n. 2, p. 19-26, abr./jun. 2010.

FELIX, Edilaine Heleodoro. A pesquisa nos grupos de pesquisa: cibernética pedagógica - Laboratório de Linguagem Digitais (LLD). 2011. Dissertação (Mestrado em Ciências da Comunicação) - Departamento de Comunicações e Artes, Escola de Comunicação e Artes, Universidade de São Paulo, São Paulo, 2011.

FERENHOF, Helio Aisenberg; FERNANDES, Roberto Fabiano. Desmistificando a revisão de literatura como base para redação científica: método SSF. Revista ACB, Florianópolis, v. 21, n. 3, p. 550-563, ago./nov. 2016.

FERRASI, Emerson Carlos Sarti. Desenvolvimento de sistema para gerenciamento de dados de pesquisa. 2013. Dissertação (Mestrado) - Faculdade de Medicina, Universidade Estadual Paulista, Botucatu, 2013.

FERRAZ, Isabela Neves. Repertório compartilhado de recursos em comunidades virtuais de prática: um estudo dos mecanismos de interação, organização e controle em grupos de pesquisa científica. Dissertação (Mestrado em Administração) - Programa de Pós-graduação em Administração, Departamento de Ciências Administrativas, Universidade Federal de Pernambuco, Recife, 2011. 
FERREIRA, Sueli Mara Soares Pinto. Redes eletrônicas e informação: abordagem Sense-Making para estudo de comportamento de usuários do Instituto de Física da USP. 1995. Tese (Doutorado em Ciências da Comunicação) - Escola de Comunicações e Artes, Universidade de São Paulo, São Paulo, 1995.

FIGUEIRA NETTO, Silvino C. A comunicação científica de redes de computadores: a experiência de pesquisadores brasileiros. 1994. Dissertação (Mestrado em Ciência da Informação) - Departamento de Ciência da Informação, Universidade Federal do Rio de Janeiro, Rio de Janeiro, 1994.

FREITAS JÚNIOR, Olival de Gusmão. Um modelo de sistema de gestão de conhecimento para grupos de pesquisa e desenvolvimento. 2003. Tese (Doutorado) - Programa de Pós-graduação em Engenharia de Produção, Universidade Federal de Santa Catarina, Florianópolis, 2003.

JANNUZZI, Celeste Aída Sirotheau Corrêa; FALSARELLA, Orandi Mina; SUGAHARA, Cibele Roberta. Sistema de informação: um entendimento conceitual para a sua aplicação nas organizações empresariais. Perspectivas em Ciência da Informação, Belo Horizonte, v. 19, n. 4, p. 94-117, out./dez. 2014.

KRAHL, Monica et al. Experiência dos acadêmicos de enfermagem em um grupo de pesquisa. Revista Brasileira de Enfermagem, Brasília, DF, v. 62, n. 1, p. 146-150, Brasília, jan./fev. 2008.

LEMOS, Claudeth da Silva; SILVA, Daniele Cariolano da; SILVA, Francisco Tiago Ribeiro; LIMA, Venícius de Sousa. Gepedes no IFCE-campus Quixadá: uma experiência de ensino-pesquisa. Revista Educação Online, Rio de Janeiro, n. 31, maio/ago. p. 143-167, 2019.

LICHTNOW, Daniel. Desenvolvimento e implementação de um protótipo de ferramenta para gestão do conhecimento em grupos de pesquisa. 2001. Dissertação (Mestrado em Ciência da Computação) - Programa de Pós-graduação em Ciência da Informação, Universidade Federal de Santa Catarina, Florianópolis, 2001.

LIMA, Karina Kühl de. Sistemas de apoio à gestão do conhecimento em redes de pesquisa: o caso Instituto Fábrica do Milênio. 2005. Dissertação (Mestrado em Engenharia da Produção) - Departamento de Engenharia, Universidade Federal de São Carlos, São Carlos, 2005.

MIRANDA, Raquel Gianolla. Tecnologias, educação e seus sentidos: o movimento de um grupo de pesquisa sobre interdisciplinaridade - GEPI. 2008. Tese (Doutorado em Educação) - Departamento de Educação, Pontifícia Universidade Católica, São Paulo, 2008. 
MOTTA, Rodrigo Patto Sá. As universidades e o regime militar: cultura política brasileira e modernização autoritária. Rio de Janeiro: Zahar, 2014.

ODELIUS, Catarina Cecília et al. Processos de aprendizagem, competências aprendidas, funcionamento, compartilhamento e armazenagem de conhecimentos em grupos de pesquisa. Cadernos EBAPE, Rio de Janeiro, v. 9, n. 1, p. 199- 220, mar. 2011.

PILATTI, Orlando. Especialização: falácia ou conhecimento aprofundado? Revista Brasileira de Pós-Graduação, [Brasília, DF], v. 3, n. 5, p. 7-26, jun. 2006. Disponível em: http://ojs.rbpg.capes.gov.br/index.php/rbpg/article/view/93/89. Acesso em: 07 jan. 2020.

PRADO, Cláudia et al. Espaço virtual de um grupo de pesquisa: o olhar dos tutores. Revista da Escola de Enfermagem da USP, São Paulo, v. 46, n. 1, p. 246-252, 2012.

RAMOS, Amauri Pereira. Construção, uso e disseminação da informação em grupos de pesquisa por meio de ambientes virtuais de colaboração. 2006. Dissertação (Mestrado) - Centro de Ciências Sociais Aplicadas, Pontifícia Universidade Católica, São Paulo, 2006.

REEDY, Joel; SCHULLO, Shauna; ZIMMERMAN, Kenneth. Marketing eletrônico: a integração de recursos eletrônicos ao processo de marketing. 2. ed. São Paulo: Bookman, 2006.

SAMPAIO, Eduardo Magalhães. Ferramentas de cooperação para o trabalho em redes de pesquisas geocientíficas. 2017. Tese (Doutorado em Geologia) - Instituto de Geociências, Universidade Federal da Bahia, Bahia, 2017.

SANTOS JÚNIOR, Victor LourençO dos. Organização da interação de pesquisadores na prática científica: um estudo de grupos de pesquisa da UFRG. 2000. Dissertação (Mestrado em Sociologia) - Instituto de Filosofia e Ciências Humanas, Universidade Federal do Rio Grande do Sul, Porto Alegre, 2000.

SANTOS, Domingos Bernardo Gomes. Aplicação de ferramentas computacionais à gestão do conhecimento científico em grupos de pesquisa. 2010. Dissertação (Mestrado em Engenharia) - Escola Politécnica, Universidade de São Paulo, São Paulo, 2010.

SANTOS, Domingos Bernardo Gomes. 0 conhecimento e a pesquisa nas nuvens: uma pesquisa social sobre a aplicação das práticas de gestão do conhecimento associadas às tecnologias de computação em nuvens em ambientes de pesquisa. 2016. Tese (Doutorado em Ciências) - Escola Politécnica, Universidade de São Paulo, São Paulo, 2016. 
SANTOS, Eduardo Tafeli Carneiro dos. Gestão eletrônica de documentos: gerenciamento do conhecimento para grupos de pesquisa. 2007. Dissertação (Mestrado em Engenharia Elétrica) - Departamento de Engenharia, Universidade Presbiteriana Mackenzie, São Paulo, 2007.

SOUZA, Maria da Paixão Neres de. As tecnologias de informação no processo de produção, legitimação e difusão do conhecimento dos pesquisadores da Embrapa. 1999. Tese (Doutorado em Ciência da Informação) - Departamento de Ciência da Informação e Documentação, Universidade de Brasília, Brasília, 1999.

TISOTT, Priscila Bresolin. Inovação em grupos de pesquisa em nanotecnologia: um estudo de caso no Rio Grande do Sul. 2015. Dissertação (Mestrado em Administração) - Departamento de Administração, Universidade de Caxias do Sul, Caxias do Sul, 2015. 